THEOREM 3. If the conditions of Theorem 2 part A are satisfied and if in addition the quantities $\psi_{1}, \psi_{2}$ and $\delta$ satisfy the inequality

$$
2 \pi>\delta\left(\csc \psi_{1}-\csc \psi_{2}\right)
$$

then the circle of convergence is not a cut for the function.

\title{
BIBLIOGRAPHY
}

1. P. Dienes, The Taylor series, Oxford, 1931.

2. E. C. Titchmarsh, The theory of functions, Oxford University Press, 1939, pp. 99-100.

LEHIGH UNIVERSITY

\section{A NOTE ON THE HILBERT TRANSFORM}

\section{LYNN H. LOOMIS}

The Hilbert transform of $f(t),-\infty<t<\infty$, is $1 / \pi$ times the Cauchy principal value

$$
\bar{f}(x)=P \int_{-\infty}^{\infty} \frac{f(t)}{t-x} d t=\lim _{\delta \rightarrow 0+} \int_{\delta}^{\infty} \frac{f(x+t)-f(x-t)}{t} d t .
$$

If $f(t) \in L_{p}, p>1$, then $\bar{f}(x) \in L_{p}$, and a considerable literature is devoted to studying the relationship of such pairs of "conjugate" functions to the theory of functions analytic in a half-plane. More to the point of the present note is a series of papers studying the Hilbert transform along strictly real variable lines $([2,3]$; further bibliography in [2]). ${ }^{1}$

Much less is known about $\bar{f}(x)$ when $f(t) \in L_{1}$. Plessner found by applying complex variable methods to the theory of Fourier series that if $f(t) \in L_{1}$ then $\bar{f}(x)$ exists almost everywhere (see $[1$, p. 145]). Besicovitch [4] proved Plessner's result using only the theory of sets, starting from his own previous real variable investigation of the $L_{2}$ transform case. S. Pollard [5] showed how Besicovitch's proof could be extended to prove the existence a.e. of the principal value of the Stieltjes integral

$$
\bar{f}(x)=P \int_{-\infty}^{\infty} \frac{d F(t)}{t-x},
$$

Received by the editors April 11, 1946.

1 Numbers in brackets refer to the bibliography at the end of the paper. 
where $F(t)$ is continuous and of bounded variation over $(-\infty, \infty)$. In general $\bar{f}(x)$ is not summable, but Kolmogoroff [6] found, using a contradiction argument, that there exists a constant $A$ such that the set where $\bar{f}(x)>M>0$ has measure at most $A\|f\| / M$, where $\|f\|=\int_{-\infty}^{\infty}|f(t)| d t$. Titchmarsh [7] was able to refine Besicovitch's existence proof so that it implied this bound, with a numerical value for $A$.

The present note contains a new direct real-variable proof of the Plessner existence theorem and the Kolmogoroff bound. In fact, this bound in a sense is the central tool for the existence proof, a device which allows for the first time the $L_{1}$ results to be obtained without recourse to the $L_{2}$ transform theory.

LEMMA 1. If $c_{i}>0$ and

$$
g(x)=\sum_{i=1}^{n} \frac{c_{i}}{x-a_{i}}
$$

then the set of points where $g(x)>M(M>0)$ consists of $n$ intervals whose total length is precisely $\left(\sum c_{i}\right) / M$. The set where $g(x)<-M$ has the same length.

Since $g\left(a_{i}-\right)=-\infty, g\left(a_{i}+\right)=\infty$ and $g^{\prime}(x)<0$ for all $x$, there are precisely $n$ points $m_{i}$ such that $g\left(m_{i}\right)=M$, and $a_{i}<m_{\imath}<a_{i+1}$, $i=1, \cdots, n-1, a_{n}<m_{n}$. The set where $g(x)>M$ thus consists of the intervals $\left(a_{i}, m_{i}\right)$ and has the total length

$$
\sum_{i=1}^{n}\left(m_{i}-a_{i}\right)=\sum_{i=1}^{n} m_{i}-\sum_{i=1}^{n} a_{i}
$$

But the numbers $m_{i}$ are the roots of the equation

$$
\sum_{i=1}^{n} \frac{c_{i}}{x-a_{i}}=M
$$

whose cross-multiplied form is

$$
\sum_{i=1}^{n} c_{i}\left[\prod_{j \not i}\left(x-a_{j}\right)\right]=M \prod_{i=1}^{n}\left(x-a_{i}\right),
$$

or

$$
M x^{n}-\left[M \sum a_{i}+\sum c_{i} x^{n-1}+\cdots=0,\right.
$$

so that

$$
\sum_{i=1}^{n} m_{i}=\sum_{i=1}^{n} a_{i}+\frac{1}{M} \sum_{i=1}^{n} c_{i}
$$


The first part of the lemma follows from (1) and (2); the proof for $g(x)<-M$ is almost identical.

LEMMA 2. Let $F(t)$ be increasing over $(-\infty, \infty)$ with finite total variation $V(F)$. If $\left(x_{j}-\delta_{j}, x_{j}+\delta_{j}\right), j=1, \cdots, n$, are disjoint intervals such that

$$
\int_{-\infty}^{x_{j}-\delta_{j}}+\int_{x j+\delta_{j}}^{\infty} \frac{d F(t)}{t-x_{j}}>M>0,
$$

then $\sum \delta_{1} \leqq 4 V(F) / M$. The same inequality is implied if the integral is less than $-M, j=1, \cdots, n$.

Let $t_{i}, i=1, \cdots, N$ be a finite subdivision including the points $x_{j}-\delta_{j}, x_{j}, x_{j}+\delta_{j}$ for $j=1, \cdots, n$, and such that the approximating Riemann sums for (3), with the integrand evaluated at the left-hand end points, remain greater than $M$. Thus, if $\Delta_{i}=F\left(t_{i+1}\right)-F\left(t_{i}\right)$,

$$
\sum_{i \notin I_{j}} \frac{\Delta_{i}}{t_{i}-y}>M
$$

for $y=x_{j}$, where the set $I_{j}$ of omitted indices is defined by

$$
\bigcup_{i \in I_{j}}\left(t_{i}, t_{i+1}\right)=\left(x_{i}-\delta_{j}, x_{j}+\delta_{j}\right) \text {. }
$$

Since the left member of (4) is an increasing function of $y$ for $x_{j}-\delta_{j}<y<x_{j}+\delta_{j}$, the inequality (4) holds for $x_{j} \leqq y<x_{j}+\delta_{j}$. For every such $y$ one of the following inequalities is therefore satisfied:

$$
\sum_{i=1}^{N-1} \frac{\Delta_{i}}{t_{i}-y}>\frac{M}{2}, \quad \sum_{i \in I_{j}} \frac{\Delta_{i}}{t_{i}-y}<-\frac{M}{2} .
$$

Applying Lemma 1 and summing over $j$, we have

$$
\sum \delta_{j} \leqq \sum_{i=1}^{N-1} \frac{2 \Delta_{i}}{M}+\sum_{j=1}^{n} \sum_{i \in I_{j}} \frac{2 \Delta_{i}}{M} \leqq \frac{4}{M} \sum_{i=1}^{N-1} \Delta_{i} \leqq \frac{4}{M} V(F) .
$$

To prove the second part of the lemma we only need to observe that the integral in (3) is less than $-M$ if and only if after replacing $F(t)$ by $-F(-t)$ and $x_{j}$ by $-x_{j}$ it is greater than $M$.

CoRollary. If $F(t)$ is of bounded variation in Lemma 2 then $\sum \delta_{j} \leqq 8 V(F) / M$.

This follows at once upon applying the lemma to the increasing and decreasing parts, $F_{1}$ and $F_{2}$, of $F$, using $V(F)=V\left(F_{1}\right)+V\left(F_{2}\right)$.

Preliminary to the theorem we remark that if $f(t)$ has the value 1 in 
$(a, b)$ and 0 elsewhere, then its Hilbert transform exists except at the two points $a$ and $b$, and has the value $\log |(x-b) /(x-a)|$. In particular the Hilbert transform of any step function exists except at a finite number of points.

THEOREM. Let $F(t)$ be of bounded variation over $(-\infty, \infty)$. Then its Hilbert-Stieltjes transform

$$
\bar{f}(x)=P \int_{-\infty}^{\infty} \frac{d F(t)}{t-x}
$$

exists almost everywhere, and, for every positive $M$, the set where $\bar{f}(x)>M$ has measure at most $16 \mathrm{~V}(F) / M$, as does the set where $\bar{f}(x)<M$.

We first prove the existence of $\bar{f}(x)$. It is sufficient to show that, given $\epsilon$, for every $x$ except in a set of measure less than $\epsilon$

$$
\left|\int_{x-\delta}^{x-\delta^{\prime}}+\int_{x+\delta^{\prime}}^{x+\delta} \frac{d F(t)}{t-x}\right| \leqq \epsilon
$$

for all sufficiently small $\delta$ and $\delta^{\prime}$. Now the absolutely continuous part of $F$ can be approximated to within $\epsilon^{\prime}$ by the integral $F_{1}$ of a step function $h, F_{1}(t)=\int_{-\infty}^{t} h(t) d t$, and the singular part of $F$ can be approximated to within $\epsilon^{\prime}$ by a singular function $F_{2}$ whose variation is confined to a closed set of measure 0 , that is, which is constant on the intervals of an open set $M$ whose complement has measure zero. Thus, taking $\epsilon^{\prime}=\epsilon^{2} / 192$, we have $F=F_{1}+F_{2}+F_{3}$, where $V\left(F_{3}\right)<\epsilon^{2} / 96$. Let $E_{\mathrm{a}}$ be the set of $x$ for which the inequality

$$
\left|\int_{x-\delta}^{x-\delta^{\prime}}+\int_{x+\delta^{\prime}}^{x+\delta} \frac{d F_{3}(t)}{t-x}\right| \leqq \frac{\epsilon}{3}
$$

fails to hold for arbitrarily small $\delta$ and $\delta^{\prime}\left(\delta^{\prime}<\delta\right)$. Then for every $x$ in $E_{e}$

$$
\left|\int_{-\infty}^{x-\Delta}+\int_{x+\Delta}^{\infty} \frac{d F_{3}(t)}{t-x}\right|>\frac{\epsilon}{6}
$$

for arbitrarily small $\Delta$. By Vitali's theorem a disjoint sequence of intervals $\left(x_{i}-\Delta_{i}, x_{i}+\Delta_{i}\right)$ satisfying (7) can be chosen so as to cover $E_{\epsilon}$ except for a set of measure 0 . Then by Lemma 2, corollary, $m\left(E_{\epsilon}\right) \leqq 2 \sum \Delta_{i} \leqq 2 \cdot 8 V\left(F_{3}\right) \cdot 6 / \epsilon<\epsilon$.

Since $F_{1}(t)$ is the integral of a step function, its Hilbert-Stieltjes transform (the Hilbert transform of $h(t)$ ) exists except at a finite number of points, which we add to $E_{e}$. Since $F_{2}(t)$ is constant on the intervals of $M$, its Hilbert-Stieltjes transform obviously exists except 
on the complement of $M$, which we add to $E_{e}$. Thus if $x$ is not in the enlarged $E_{\epsilon}$ there is a $\Delta$ such that, for all $\delta$ and $\delta^{\prime}$ less than $\Delta,(6)$ holds for $F_{1}, F_{2}$ and $F_{3}$, and hence (5) holds, as was to be proved.

The second part of the theorem follows immediately from Lemma 2, corollary, where the intervals $\left(x_{j}-\delta_{j}, x_{j}+\delta_{j}\right)$ are chosen by Vitali's theorem to cover almost entirely the set where $\bar{f}(x)>M(<-M)$, so that the measure of this set is not greater than $2 \sum \delta_{i} \leqq 16 V(F) / M^{\prime}$.

Corollary. If $0<p<1$ and $p+q>1$, then $|\bar{f}(x)|^{p} /(1+|x|)^{q} \in L_{1}$.

This follows immediately from the fact that the decreasing function on $(0, \infty)$ which is equimeasurable with $|\bar{f}(x)|$ is dominated by $K / x$.

In case $F(t)$ is singular and increasing, it can be shown with little difficulty that the constant 16 can be replaced by 1 , and this is best possible since $1 / x$ itself is the Hilbert-Stieltjes transform of the function $F(t)$ which is 1 when $t<0$ and 0 when $t \geqq 0$. This is probably the correct value of the constant in the general case.

\section{BIBLIOGRAPHY}

1. A. Zygmund, Trigonometrical series, Warsaw, 1935.

2. G. H. Hardy, On Hilbert transforms, Quart. J. Math. Oxford Ser. vol. 3 (1932) pp. 102-112.

3. J. Cossar, On conjugate functions, Proc. London Math. Soc. vol. 45 (1939) pp. 369-381.

4. A. S. Besicovitch, On a general metric property of summable functions, J. London Math. Soc. vol. 1 (1926) pp. 120-128.

5. S. Pollard, Extension to Stieltjes integrals of a theorem due to Plessner, J. London Math. Soc. vol. 2 (1926) pp. 37-41.

6. A. Kolmogoroff, Sur les fonctions harmonique conjuguées at les series de Fourier, Fund. Math. vol. 7 (1925) pp. 24-29.

7. E C. Titchmarsh, On conjugate functions, Proc. London Math. Soc. vol. 29 (1929) pp. 49-80.

\section{HARVARD UNIVERSITY}

